\title{
Toward a Neurobiological Model of Cue-Induced Self-Control in Decision Making: Relevance to Addiction and Obesity
}

\author{
Agnes J. Jasinska, ${ }^{1}$ Anand Ramamoorthy, ${ }^{2}$ and Christopher M. $\mathrm{Crew}^{3}$ \\ ${ }^{1}$ Department of Psychology, University of Michigan, Ann Arbor, Michigan 48109, 2Department of Experimental Psychology, Ghent University, 9000 Ghent, \\ Belgium, and ${ }^{3}$ Department of Psychology, Columbia University, New York, New York 10027 \\ Review of Hare et al.
}

Self-control is a key aspect of adaptive decision-making. Self-control allows us to pursue a deliberate, long-term goal (for instance, to be healthy) by overcoming more automatic and immediate stimulus-response tendencies that conflict with that goal (to eat a sugary snack). Conversely, impaired self-control in decision-making has been linked to addiction and obesity (for review, see Li and Sinha, 2008; Feil et al., 2010), highlighting the enormous impact of this issue on public health as well as the importance of research on the neurobiological mechanisms of selfcontrol in decision-making.

Neuroimaging evidence suggests that the ventromedial prefrontal cortex (VMPFC) is involved in computing the overall subjective value (a stimulus value, also referred to as a goal value) of specific behavioral choices and in using this value to bias subsequent decision-making (for review, see Kable and Glimcher, 2009; Rangel and Hare, 2010). A parallel line of evidence points to the VMPFC, as well as the dorsolateral prefrontal cortex (DLPFC) and the inferior frontal

Received Aug. 19, 2011; revised Sept. 25, 2011; accepted Sept. 26, 2011. We thank our mentors, Drs. Emily Falk, Thad Polk, Tom Verguts, and Geraldine Downey, for helpful comments. We also thank the organizers, speakers, and fellow attendees of the Summer Institute of Cognitive Neuroscience 2011, where this collaboration started, for a very enriching and inspiring experience.

Correspondence should be addressed to Agnes J. Jasinska, 530 Church Street, Ann Arbor, MI 48109. E-mail: jasinska@umich.edu.

DOI:10.1523/JNEUROSCI.4477-11.2011

Copyright $\odot 2011$ the authors $\quad 0270-6474 / 11 / 3116139-03 \$ 15.00 / 0$ gyrus (IFG), as key sites of self-control processes, including both inhibitory control and emotion regulation (for review, see Aron et al., 2004; Ochsner and Gross, 2005; Li and Sinha, 2008).

However, the neurobiological mechanisms linking self-control processes with value-based decision-making are still incompletely understood. While evidence suggests that the VMPFC is involved both in self-control and in value-based decisions, it is unclear whether this region integrates self-control signals and value signals to guide behavioral choices. Furthermore, the influence of the DLPFC and IFG on the value signals in the VMPFC is not well understood. Finally, although the valuation circuitry is activated by external stimuli, what activates the self-control circuitry is unknown.

A recent study by Hare et al. (2011) sheds light on these questions. Hare et al. (2011) used functional MRI to examine the impact of health cues on self-control processes in the DLPFC/ IFG and value signals in the VMPFC during dietary choices. The subjects were healthy, nondieting, hungry subjects. On each trial, subjects saw a food item and decided whether or not they wanted to eat it. One trial per subject was chosen at random and subjects received that food item at the end of the experiment. The subjective stimulus value at the time of decision was measured with a four-point scale ranging from "strong no" to "strong yes." The food items were classified into four categories based on subjects' ratings of health and taste attributes: unhealthy-untasty, healthy-untasty, unhealthy-tasty, and healthy-tasty. The trials with healthy-untasty options and unhealthy-tasty options were considered self-control trials. In addition, the selfcontrol processes were manipulated with three types of attentional cues. In the health condition (HC), subjects received health cues instructing them to focus on the healthiness of the food before making a decision; in the taste condition, subjects were instructed to focus on the taste of the food; and in the natural condition (NC), which served as a baseline, subjects were asked to consider each food item naturally.

The main hypothesis of the study was that health cues should modulate the impact of health attributes of specific foods on both brain and behavioral correlates of dietary decision-making. More specifically, Hare et al. (2011) hypothesized that health cues would improve dietary choices by engaging self-control processes in the DLPFC and IFG regions and by increasing the contribution of health attributes to the value signals in the VMPFC.

As in previous studies (Hare et al., 2009), activation of the VMPFC was positively correlated with the subjective stimulus value of each food option across all task conditions. As shown with conjunction analysis, the activity in an overlapping 

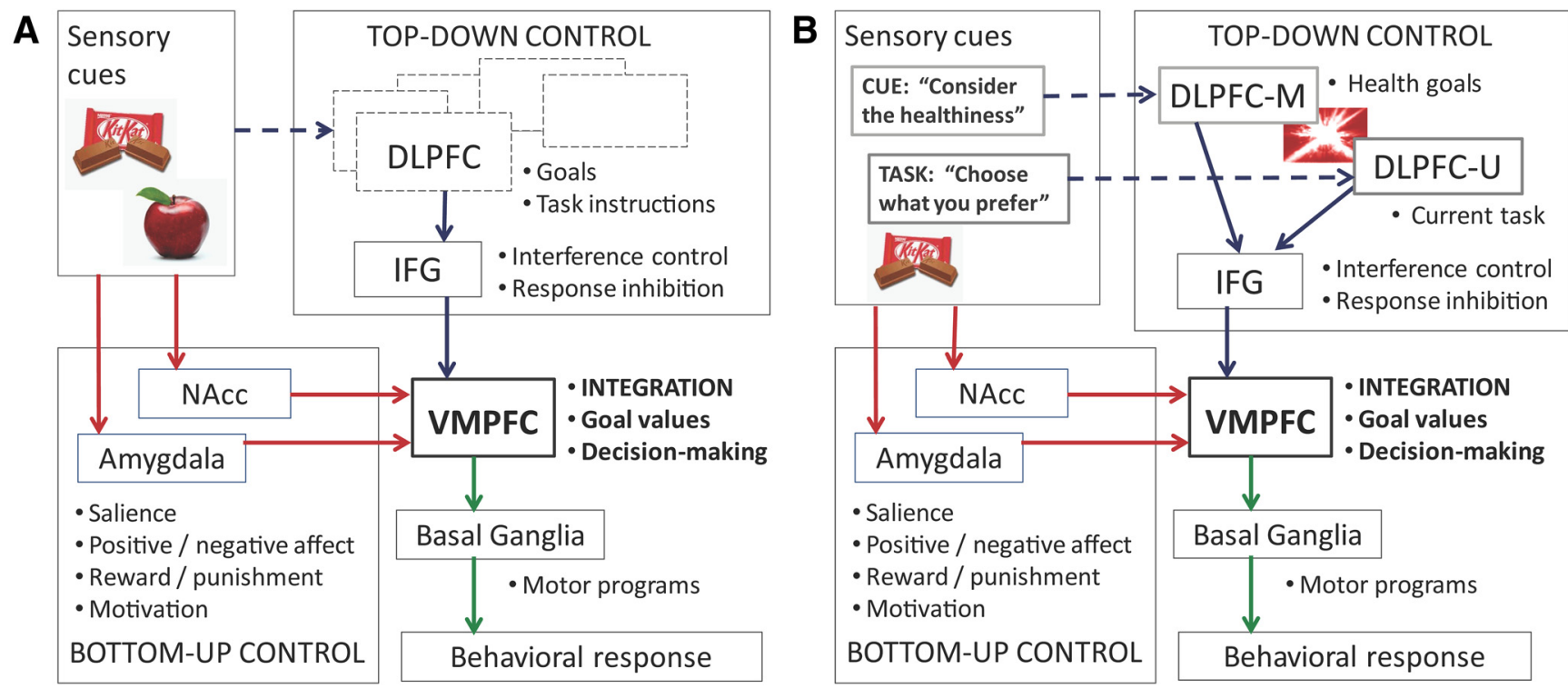

Figure 1. Neurobiological mechanism of cue-induced self-control during dietary decision-making. $\boldsymbol{A}$, The general DLPFC-IFG-VMPFC mechanism underlying self-control during dietary choice. Different external sensory cues activate both the top-down and bottom-up control streams. The DLPFC represents abstract goals and task instructions. These distributed representations in the DLPFC may be activated both endogenously and via external sensory cues. The DLPFC then signals to the IFG. The IFG resolves conflict between any competing goal and task representations in the DLPFC by inhibiting the signal from the losing representation. The IFG signals to the VMPFC. The VMPFC integrates multiple stimulus attributes, as signaled by both the top-down and bottom-up control streams, into an overall goal value (or stimulus value) that biases the behavioral choice. $\boldsymbol{B}$, The specific attentional-modulation paradigm used by Hare et al. (2011) induces an additional conflict between two different representations in the DLPFC. The DLPFC-M represents a pre-existing goal to be healthy. This goal gets reactivated by health cues. In contrast, the DLPFC-U represents the current task instructions, which ask subjects to respond as they prefer (i.e., based on taste rather than health considerations), despite attentional cues. Thus, the goal to be healthy (DLPFC-M) and the current task instructions (DLPFC-U) are partially in conflict, graphically represented by an electric current connecting the DLPFC-M and DLPFC-U. NAcc, nucleus accumbens.

VMPFC region also positively correlated with subjective health and taste ratings, suggesting that the stimulus value signal incorporated both health and taste attributes of each food option.

But do health cues engage self-control processes in the DLPFC and IFG, and do these self-control processes then modulate the stimulus value in the VMPFC and improve dietary choices? As hypothesized, Hare et al. (2011) found that compared with choose-naturally cues, health cues preferentially activated the left IFG (BA 47/46) and two subregions of the left DLPFC: one modulated by health ratings (DLPFC-M; BA 9) and one unmodulated by health ratings (DLPFC-U; BA 8/9). These data support the notion that health cues serve to engage selfcontrol processes during dietary choice. Importantly, the impact of health cues on the stimulus value signal in the VMPFC was mirrored by the impact of these health cues on actual behavioral choices. At the neural level, health cues increased the weight of healthiness on the stimulus value of each food item in the VMPFC; at the behavioral level, the same health cues improved the likelihood that subjects would choose healthy-untasty snacks and refrain from choosing unhealthy-tasty foods.

Even more interesting are the results of functional connectivity analyses reported by Hare et al. (2011). In their previous study, Hare et al. (2009) used a similar food-choice task but without attentional cues in subjects who were actively dieting. The authors found that the DLPFC (IFG/BA 9) modulated the stimulus value in the VMPFC through the IFG (BA 46), as shown with psychophysiological interaction. This was particularly true in subjects classified as efficient self-controllers who rejected most of the unhealthy-tasty foods. Thus, the results supported a three-node circuit underlying self-control during dietary decisionmaking, DLPFC $\rightarrow$ IFG $\rightarrow$ VMPFC.

In contrast, in the most recent study (Hare et al., 2011), the circuit tested with dynamic causal modeling analyses contained four nodes: the VMPFC, IFG, and two distinct DLPFC nodes (DLPFC-M and DLPFC-U). The bidirectional coupling between VMPFC, IFG, and DLPFC-M increased at the time of decision regardless of the cue condition; in contrast, the coupling from DLPFC-U to VMPFC decreased during choice. In addition, the coupling from DLPFC-U to IFG was negatively modulated by DLPFC-M, while the coupling from DLPFC-M to VMPFC was positively modulated by DLPFC-U.

What are the roles of the two DLPFC subregions? One possibility is graphically represented in Figure 1. Even in subjects who are not actively dieting, the DLPFC-M encodes a goal to be healthy. This goal representation gets reactivated by health cues and triggers a cascade of top-down self- control processes that modulate decisionmaking. Specifically, in response to health cues, the DLPFC-M signals to the IFG, which signals to the VMPFC to increase the weight of health attributes when making a decision about a specific food item. In contrast, and of particular importance in the attentional-modulation paradigm used by Hare et al. (2011), the DLPFC-U encodes task instructions, including the important provision that subjects should always choose the food item they prefer, regardless of the attended attribute. Thus, the DLPFC-M and DLPFC-U are partially in conflict: the goal to be healthy (DLPFC-M) dictates that one always chooses a healthy option, while the task instructions to choose as one prefers (DLPFC-U) are ambiguous with respect to the pre-existing goal to be healthy and partially undermine it. This explains why the DLPFC-M inversely modulates the impact of the DLPFC-U on the IFG (and thus also VMPFC) at time of decision. It also explains why the DLPFC-U was identified in the HC-NC contrast in the first place: the conflict between the DLPFC-U and DLPFC-M should be the largest in response to health cues. We further propose that this conflict is resolved at the level of the IFG, which then signals to the VMPFC to modulate the stimulus value accordingly. More generally, while the VMPFC integrates both top-down and bottom-up influences into an overall stimulus value that 
guides behavioral choice, the IFG serves as a key inhibitory gate for the top-down control stream before it reaches the VMPFC, consistent with the extensive literature on the role of IFG in interference resolution and response inhibition (Aron et al., 2004).

The key novel finding of the study by Hare et al. (2011) is that the brain processes associated with voluntary self-control can also be triggered by external cues. Specifically, the authors demonstrate that simple verbal cues that call attention to specific attributes of a food item can trigger selfcontrol processes in the DLPFC and IFG, and these control processes then modulate the stimulus value of this item in the VMPFC and thus bias behavioral choice. These results effectively blur the distinction between endogenously generated and exogenously triggered control of behav- ior. Thus, when self-control is required to choose a healthy-untasty snack and reject an unhealthy-tasty one, an efficient selfcontroller may activate such self-control on his own, while an individual with impaired endogenous self-control may be aided by external cues. Future studies aimed at characterizing the specific contributions of the DLPFC, IFG, and VMPFC will not only inform our understanding of the neurobiological mechanisms of self-control during health-related decisions, but could also lead to novel treatments for addiction and obesity.

\section{References}

Aron AR, Robbins TW, Poldrack RA (2004) Inhibition and the right inferior frontal cortex. Trends Cogn Sci 8:170-177.

Feil J, Sheppard D, Fitzgerald PB, Yücel M, Lubman DI, Bradshaw JL (2010) Addiction, compulsive drug seeking, and the role of frontostriatal mechanisms in regulating inhibitory control. Neurosci Biobehav Rev 35:248-275.

Hare TA, Camerer CF, Rangel A (2009) Selfcontrol in decision-making involves modulation of the vmPFC valuation system. Science 324:646-648

Hare TA, Malmaud J, Rangel A (2011) Focusing attention on the health aspects of foods changes value signals in vmPFC and improves dietary choice. J Neurosci 31:1107711087.

Kable JW, Glimcher PW (2009) The neurobiology of decision: consensus and controversy. Neuron 63:733-745.

Li CS, Sinha R (2008) Inhibitory control and emotional stress regulation: neuroimaging evidence for frontal-limbic dysfunction in psychostimulant addiction. Neurosci Biobehav Rev 32:581-597.

Ochsner KN, Gross JJ (2005) The cognitive control of emotion. Trends Cogn Sci 9:242-249.

Rangel A, Hare T (2010) Neural computations associated with goal-directed choice. Curr Opin Neurobiol 20:262-270. 